\title{
Index of Contributors
}

A

Abdin MZ, 45

Abliz Z, 77

Amarakoon T, 377

Ando M, 58

B

Bagul MS, 149

Bao SX, 195

Bay BH, 35, 223, 259, 428

C

CaiCH, 188

Campbell BC, 231

Cao T, 223

Che CT, 134

Chen G, 109

Chen GY, 81

Chen JP, 252

Chen XH, 109

Chen ZT, 27

Chou V, 365

Chua YRR, 418

Clayton A, 439

D

Dai JG, 58

De Silva R, 377

Du HY, 428

Duan W, 347, 365

F

Fong WF, 81

Foong WC, 223

Forgo $\mathrm{P}, 85$

Fu GM, 77

G

Gibbs T, 1
Gong ZN, 335

GuX, 324

Guo D, 58

GuoZY, 142

H

Hamburger M, 206

Hashimoto M, 312

Hohmann J, 85

Hsu A, 418

$\mathrm{Hu} X, 347$

Huang HQ, 195

Huang SH, 347, 377

Huang Y, 142

I

Islam $\mathrm{N}, 312$

J

JiLL, 324

K

Khalequzzaman M, 312

Kim JH, 231

Kövér KE, 85

L

Lan WJ, 188

Lee YK, 259

Li B, 77

LiGY, 81

LiHJ, 188

$\mathrm{Li} \mathrm{YH,} 428$

$\operatorname{Lim} \mathrm{D}, 35$

Lin YC, 142, 188

Ling LJ, 252

Liu P, 27

Liu T, 101

Liu ZD, 27

Low LS, 365 


\section{M}

Mahendran R, 259

Mahoney N, 231

Mao JC, 347

Meng QH, 27

Molyneux RJ, 231

O

Okuno T, 312

Olivo M, 428

On T, 347

Ouyang HW, 223

P

Pan K, 134

Parveen SA, 312

$\mathbf{R}$

Rajani M, 149

Reese PB, 71

Rose P, 267

S

She ZG, 142

Shi PH, 252

Su ZG, 101

Sun J, 347

$\mathbf{T}$

Tan BKH, 418

Tang MJ, 77

Teng JW, 177

W

Wang M, 324

Wong WH, 365

Wu BW, 195

Wu J, 127

WuXY, 142

Wu YJ, 347

Wu ZL, 285
X

Xia GL, 285

Xie LP, 285

Xu GL, 335

Y

Yang J, 27, 127

Yang JY, 252

Yang L, 58

Yang RL, 285

Yang Y, 285

Yee TH, 296

Yip GW, 35, 223

Yong YJ, 259

Young RE, 439

Yu SS, 77

Z

Zeng Z, 177

Zhang DJ, 127

Zhang GF, 101

Zhang QH, 324

Zhang RQ, 285

Zhang S, 127

Zhang WH, 134

Zhang WY, 252

Zhou SF, 365

Zhou YP, 188

Zhu GY, 81

Zhu YZ, 347, 365

Zou XH, 223 